\title{
Disponibilidad de biomasa y contenido de proteína cruda de Hyparrhenia rufa y Panicum maximun cv Tanzania asociadas con Leucaena leucocephala y Gliricidia sepium en sistema de pasturas en callejones
}

\section{Availability of biomass and crude protein contents of Hyparrhenia rufa and Panicum maximum cv Tanzania associated with Leucaena leucocephala and Gliricidia sepium in pasture alley cropping system}

\author{
Álvaro Jose González Martínez ${ }^{1}$, Joel Rojas Hernández², Francisco José Chavarría-Ñamendiª, Ronaldo Martín Jiménez \\ Gómez $^{4}$
}

'MSc. Agroforestería Tropical. ajgonzalez75@gmail.com / ${ }^{2}$ MSc. Producción Animal Sostenible. joelrojas3@yahoo.com / ${ }^{3}$ MSc. Conservación y Manejo de Vida Silvestre /4 Ingeniero Agrónomo. ronaldo.jimenez@hotmail.com

Universidad Internacional Antonio de Valdivieso (UNIAV), Rivas, Nicaragua.

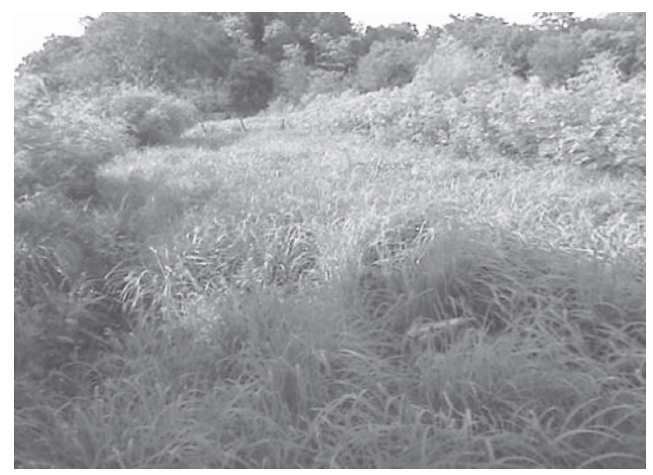

\section{RESUMEN}

Se evaluó el efecto del asocio de H. rufa y P. maximun cv Tanzania con leucaena y madero negro en la disponibilidad de biomasa y contenido de proteína cruda (PC). El estudio se desarrolló en la finca Santa María, La Chocolata, Rivas. El área fue de 2 579,2 m2 dividida en dos parcelas con: tres hileras dobles de madero negro y dos de leucaena con una distancia de $7 \mathrm{~m}$ entre callejón, $1 \mathrm{~m}$ entre surco y 0,5 m entre plantas. La evaluación se realizó entre 2009 y 2010 con siete ciclos de pastoreo y frecuencia de 48 a 53 días de descanso. La disponibilidad y composición botánica se realizó con la técnica de doble muestreo BOTANAL un día antes del pastoreo. Se definió escalas del 1 al 5 con base al porcentaje de cobertura, altura, relación tallo-hoja (pasto); diámetro de copa, altura y número de rebrotes (leguminosa). Se cortó el pasto a $20 \mathrm{~cm}$ y a $80 \mathrm{~cm}$ las leguminosas. Para la materia seca se tomó una muestra de $200 \mathrm{~g}$ y se secó por $72 \mathrm{~h}$ a $60^{\circ} \mathrm{C}$. Se realizó un ANOVA para medidas repetidas y una regresión lineal. Se encontró que $H$. rufa tiene mayor altura en macolla con $95,4 \mathrm{~cm}$ superando en más del 11\% a Tanzania, no obstante este último superó en diámetro de macolla en más del 16\% a $H$. rufa. Tanzania contiene $24 \%$ más de biomas que $H$. rufa y con el aporte de biomasa de la leguminosa esta diferencia es del $22.2 \%$. Leucaena supera en $90 \%$ y $22 \%$ en disponibilidad de biomasa a madero en ambas pasturas. En el contenido de PC, Tanzania es superior $34 \%$ y $2,3 \%$ en la parte comestible y no comestible respectivamente. De igual manera leucaena superó 13 y $18 \%$ a madero negro en las dos pasturas.

Palabras clave: Pasturas en callejones, proteína cruda, disponibilidad de biomasa, composición botánica, leguminosas arbóreas.
ABSTRACT
It was evaluated the effect of the partnership of $H$. rufa and $P$. maximun cv Tanzania with L. leucocephala and G. sepium in the availability of biomass and crude protein content (PC). The study was carried out in the property Santa María, La Chocolata, Rivas. The area was of $2579.2 \mathrm{~m} 2$ divided into two plots with: three double rows of black and two G. sepium of L. leucocephala, with a distance of $7 \mathrm{~m}$ between alley, furrow between $1 \mathrm{~m}$ and $0.5 \mathrm{~m}$ between plants The evaluation was carried out between 2009 and 2010 with seven cycles of grazing and frequency of 48 to 53 days of rest. The availability and botanical composition was made with the technique of double-sampling BOTANAL a day before grazing. Defined scales from 1 to 5 based on the percentage of cover, height, relationship stem-leaf (grass); cup diameter, height and number of sprouts (legume). The grass is short to $20 \mathrm{~cm}$ and $80 \mathrm{~cm}$ the legumes. For the dry matter is took a sample of $200 \mathrm{~g}$ and are dry for $72 \mathrm{~h}$ at $60^{\circ} \mathrm{C}$. It is performed an ANOVA for repeated measures and a linear regression. It was found that $\mathrm{H}$. rufa is higher on tillering with 95.4 surpassing by more than $11 \%$ to Tanzania, however this last exceeded in diameter of tillers in over $16 \%$ to H. rufa. Tanzania contains $24 \%$ more than biomes that $H$. rufa and with the contribution of biomass of the legume is difference is $22.2 \%$. L. leucocephala exceeds $90 \%$ and $22 \%$ in availability of biomass to $G$. sepium in both pastures. In the contents of PC, Tanzania is above than $34 \%$ and $2.3 \%$ in the edible and non-edible part respectively. Similarly L. leucocephala overcame 13 and $18 \%$ to G. sepium in two pastures.

Keywords: Pastures alley, protein crude, biomass disponibility, botanical composition, leguminous trees.
Recibido: 12 de enero 2017

Aceptado: 10 de abril del 2017

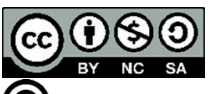

Los artículos de la revista La Calera de la Universidad Nacional Agraria, Nicaragua, se comparten bajo términos de la licencia Creative Commons: Reconocimiento, No Comercial, Compartir Igual. Las autorizaciones adicionales a las aquí delimitadas se pueden obtener en el correo freddy.aleman@ci.una.edu.ni Copyright 2017. Universidad Nacional Agraria 


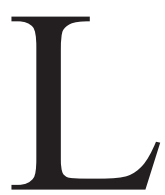

a productividad de los sistemas pecuarios ha tenido una tendencia a declinar, como consecuencia de la implementación de sistemas extensivos y de la incorporación de suelos de menor fertilidad, en los que se plantaron especies no adaptadas, generando mayor proporción de pasturas degradadas y poco productivas (Pezo et al 1992). Por otra parte, estas pasturas contienen solamente gramíneas tropicales, las cuales se caracterizan por tener bajo contenido de proteína cruda y baja digestibilidad (Bolívar y Montenegro 2001). Además, son deficientes en minerales esenciales para el ganado ya que dependen de la interacción de varios factores como el suelo, especie de planta, estado de madurez, rendimiento, clima y manejo de la pastura (McDowell et al 1984).

Se ha estimado que alrededor de la mitad de las pasturas están degradadas en algún grado (Szott et al. 2000), entendiéndose como "degradación" a la reducción temporal o permanente de su capacidad productiva (Stocking y Murnaghan 2001). Esta es causada por varias razones o combinaciones de ellas: la introducción de especies forrajeras no adaptadas a una determinada región, el mal manejo de las pasturas (como el sobrepastoreo), la compactación del suelo, la erosión y reducción de la fertilidad del suelo (Ruiz, 1983; Spain y Gualdrón, 1991; Giraldo y Bolívar, 1999). Aspectos que reducen aún más la productividad de la ganadería en la región (Pezo e Ibrahim 1999).

En Nicaragua las pasturas son manejadas en forma extensiva que sumado al mal manejo han contribuido relativamente a la baja productividad y calidad nutritiva de los pastos. Uno de los elementos nutricionales que se relaciona con la degradación de las pasturas es la deficiencia del nitrógeno. En este sentido, las pastura en callejones incluyen entre sus componentes a leguminosas arbóreas que proporcionan forraje rico en proteína y mejoran la fertilidad del suelo a través de la fijación de nitrógeno y la incorporación de mulch que forma una capa gruesa de materia orgánica que protege al suelo de erosiones eólicas e hídricas; y que además, permite un intercambio superficial de nutrientes (Pezo e Ibrahim 1999)

Dado que la incorporación de leguminosas forrajeras en pasturas ha sido señalado como una opción prometedora para revertir los procesos de degradación del suelo, además que permite aumentar la cantidad y calidad de la biomasa forrajera, este estudio se propuso evaluar el efecto del asocio de pastos (Hyparrenia rufa y Panicum maximun cv Tanzania) con plantas arbustivas (Leucaena leucocephala y Gliricidia sepium) en la producción de fitomasa de ambos componentes.

\section{MATERIALES Y MÉTODOS}

Ubicación. La investigación se desarrolló en la finca Santa María, propiedad de la Universidad Internacional de Agricultura y Ganadería, ubicada en la comunidad La Chocolata, municipio de Rivas, situada entre las coordenadas $11^{\circ}$ $41^{\prime}$ Latitud Norte; $85^{\circ} 83^{\prime}$ 'Longitud Oeste. Las condiciones agroecológicas de la zona son: temperatura media anual de $27{ }^{\circ} \mathrm{C}$, precipitaciones media anual de $1450,3 \mathrm{~mm}$, período canicular del 15 de julio - 15 de agosto, altitud de $70 \mathrm{msnm}$, humedad relativa entre $72 \%-86 \%$, velocidad del viento de noviembre - abril es de 3,7 m seg- ${ }^{1}$ y de mayo - octubre de $2,4 \mathrm{~m}$ seg-1, topografía con pendientes del $3-5 \%$, suelos de francos a francos arcillosos (INETER: http://www.ineter. gob.ni/). Es una zona cálida y seca (Salas 1993).

Diseño del área de estudio. El área del estudio fue de 2 $579,2 \mathrm{~m}^{2}$ dividido en dos parcelas de $1294,3 \mathrm{~m} 2(46,4 \mathrm{~m}$ x 27,9m) y $1284,9 \mathrm{~m}^{2}(46,1 \mathrm{~m}$ x $27,9 \mathrm{~m})$ respectivamente. Años anteriores fue un sistema de cultivos en callejones, con plantas leguminosas (L. leucocephala y G. sepium). Para el propósito de este estudio se realizó recuento y resiembra de plantas forrajeras. Cada parcela contó con tres hileras dobles de G. sepium y dos de L. leucocephala. La distancia entre las hileras dobles de las leguminosas arbóreas (ancho de callejón) fue de $7 \mathrm{~m}$ y el marco de siembra de las especies fue de 1 x $0,5 \mathrm{~m}$. Con el propósito de homogenizar la altura de las dos especies arbóreas, se realizó poda a $40 \mathrm{~cm}$ de altura un mes antes del establecimiento de los pastos.

El establecimiento de las pasturas en los callejones se realizó en los meses de julio - agosto 2008. La preparación del suelo fue mecanizada con un pase de arado y dos de gradas. La siembra de los pastos se realizó en el mes de agosto, al voleo para $H$. rufa y en surcos para $P$. maximun $c v$ Tanzania con distancia de $70 \mathrm{~cm}$, en su respectivo potrero. Se aplicó fertilizante completo a razón de $1,5 \mathrm{qq} \mathrm{mz}^{-1}$ y urea al $46 \%$ de $1 \mathrm{qq} \mathrm{mz}^{1}$, en dos momentos, a los 20 y 45 días de emergidos los pastos. El mantenimiento de pasturas y las arbustivas consistió en podas de uniformidad una vez finalizado el periodo de pastoreo a una altura de 20 a 30 y $80 \mathrm{~cm}$ respectivamente.

Periodo de estudio. El levantamiento de información se realizó a partir de septiembre de 2009 hasta agosto 2010, realizándose siete ciclos de pastoreo, tres de ellos en verano y cuatro en invierno con una frecuencia entre 48 a 53 días de descanso y un periodo de ocupación de 1 a 3 días según la época del año (cuadro 1).

Cuadro 1. Período de evaluación, días de descanso y época

\begin{tabular}{|c|c|c|c|c|c|c|}
\hline \multirow[b]{2}{*}{$\begin{array}{l}\text { Ciclo de } \\
\text { pastoreo }\end{array}$} & \multicolumn{3}{|c|}{ Pastura P. máximum cv Tanzania } & \multicolumn{3}{|c|}{ Pastura H. rufa } \\
\hline & Fecha & $\begin{array}{c}\text { Días de } \\
\text { descanso }\end{array}$ & Época & Fecha & $\begin{array}{l}\text { Días de } \\
\text { descanso }\end{array}$ & Época \\
\hline I & $21 / 09 / 09$ & 50 & \multirow{3}{*}{ Lluviosa } & $17 / 09 / 09$ & 49 & \multirow{3}{*}{ Lluviosa } \\
\hline II & $12 / 11 / 09$ & 48 & & $11 / 11 / 09$ & 51 & \\
\hline III & $28 / 01 / 10$ & 48 & & $27 / 01 / 10$ & 46 & \\
\hline IV & $19 / 03 / 10$ & 49 & \multirow[t]{2}{*}{ Seca } & $18 / 03 / 10$ & 48 & \multirow[t]{2}{*}{ Seca } \\
\hline $\mathrm{V}$ & $12 / 05 / 10$ & 53 & & $11 / 05 / 10$ & 53 & \\
\hline VI & $07 / 07 / 10$ & 52 & \multirow{2}{*}{ Lluviosa } & 07/07/10 & 51 & \multirow{2}{*}{ Lluviosa } \\
\hline VII & $31 / 08 / 10$ & 49 & & $31 / 08 / 10$ & 48 & \\
\hline Promedio / ciclo & & 49.9 & & & 49.4 & \\
\hline
\end{tabular}


Diseño experimental. El muestreo de la producción de biomasa se realizó bajo un estudio de caso, es decir el levantamiento y registro de la información de cada variable se ejecutó en el 100\% del área total de cada parcela, comparándose estadísticamente como parcelas apareadas. Para esto se realizó un ANOVA para medidas repetidas en el tiempo bajo un diseño en parcelas divididas (Morales et al., 2009), donde los tratamientos en la parcela principal correspondieron a pastos y las especies arbóreas a las subparcelas.

Disponibilidad de forraje (Biomasa) y composición botánica de la pastura. Para la disponibilidad y composición botánica de las pastura se aplicó la técnica del BOTANAL (Haydock y Shaw 1975; Tothill et al., 1992, Hargreaves y Kerr 1992). Realizado un día antes que los animales entraran al pastoreo. Para cada componente del sistema (pasto y arbustos), se establecieron escalas de producción de 1 a 5 , donde 1 se consideró a sitios de menor producción y 5 a los de mayor producción. Para definir las escalas se midió el porcentaje de cobertura, la altura y la relación tallo - hoja para pastos y en las arbustivas se consideraron la altura, diámetro de copa y número de rebrotes.

Se utilizaron tres muestras destructivas en cada escala para determinar la biomasa real por especie, separando el material comestible (hojas verdes y tallos tiernos) y el no comestible (hojas secas y tallos lignificados o gruesos). La altura del corte se realizó a $20 \mathrm{~cm}$ para las dos especies de pastos y $80 \mathrm{~cm}$ para las leñosas arbustivas. Se registraron los pesos frescos y se tomó una muestra de $200 \mathrm{~g}$ para llevarla al laboratorio y secarla en un horno a $60^{\circ} \mathrm{C}$ por 72 horas.
$M v=$ es el promedio de las muestras visuales (usando la escala de 1 a 5).

$M r=$ el promedio de los niveles en la escala real que va de 1 a 5 ; es decir, tiene el valor de 3.

$\mathrm{b}=$ coeficiente de regresión entre observaciones visuales y valores obtenidos para la cantidad de materia seca en las muestras reales tomadas

Calidad nutritiva del forraje en oferta (PC). Para estimar la proteína cruda (PC) de los pastos y arbustivas, se trituraron las muestras secas en molino Wiley, con tamaño de partículas de $1 \mathrm{~mm}$, determinando por el método de referencia de Kjeldhal el contenido de proteína (6,25 x N) (Bateman 1970, Romero 1997, Verdecia et al. 2014). Esto se realizó en el laboratorio de suelos y agua de la Universidad Internacional de Agricultura y Ganadería.

\section{RESULTADOS Y DISCUSIÓN}

En promedio, durante el período de estudio la biomasa forrajera disponible total de los pastos asociado con las leguminosas arbóreas, la pastura $P$. maximun cv Tanzania (3.1 t MS $\mathrm{ha}^{1}$ ) fue superior en más del $22 \%$ a la pastura $H$. rufa. Este dato es similar a lo encontrado por Lara (2005) quien estimó 3.47 t MS ha a los 42 días como monocultivo. Es importante señalar que después del pastoreo se observó mayor cantidad de material no comestible en $H$. rufa que en $P$. máximum, debido a que los animales consumen durante el pastoreo este tipo de material compuesto en su mayoría por hojas y que en H. rufa corresponde a tallos (cuadro 2).

Cuadro 2. Disponibilidad de biomasa kg MS ha-1 Pastura P. maximun cv Tanzania y H. rufa

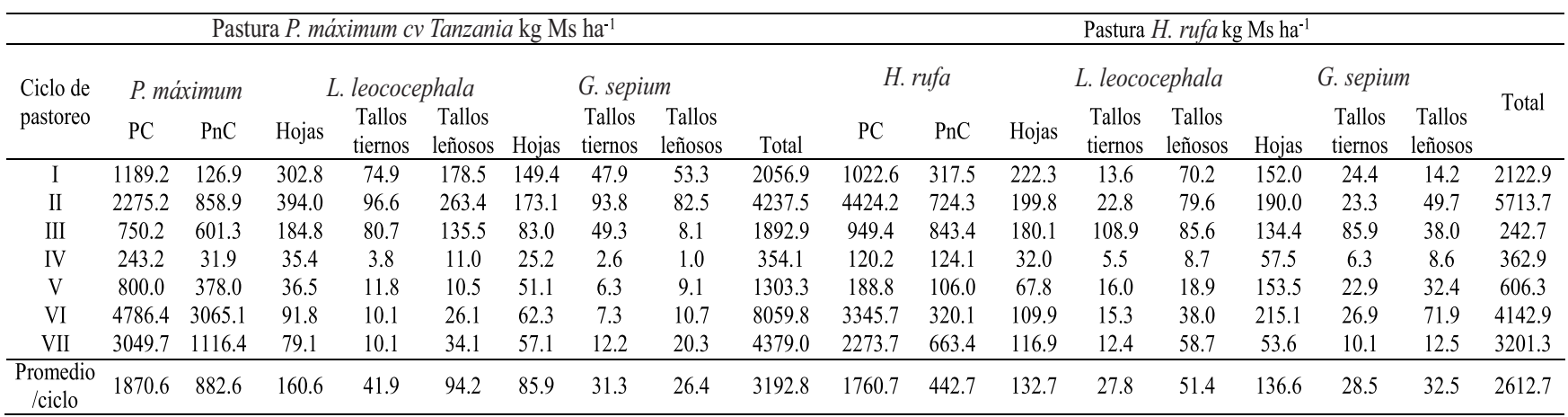

$\mathrm{PC}=$ Parte comestible; $\mathrm{PnC}=$ Parte no comestible

En los pastos se utilizó el cuadrante de $1 \mathrm{~m}$ x $1 \mathrm{~m}$ para la realización de las 60 observaciones visuales por aparto, para determinar la composición botánica y la escala correspondiente. En las arbustivas también se aplicaron 60 observaciones visuales. Con los promedios de producción de materia seca de cada escala real y las 60 escalas visuales se realizó una regresión lineal para determinar la biomasa de la pastura y arbustivas en $\mathrm{t} \mathrm{ha}^{-1}$ (Tothill et al., 1992).

Para ello se utilizó la ecuación de regresión $y=x+b(M v-M r)$ Donde:

$\mathrm{y}=$ producción estimada de materia seca por $1 \mathrm{~m}^{2}$.

$\mathrm{X}=$ producción promedio en base seca de muestras reales de 1 a 5 .
En la época seca, el promedio de biomasa forrajera total de las pasturas en $P$. máximum cv Tanzania fue de 1.2 t MS ha- ${ }^{1}$ superior en más del $4 \%$ a la pastura de $H$. rufa, este dato es similar a lo estimado por Verdacea et al (2001) quien determinó $1.02 \mathrm{t} \mathrm{MS} \mathrm{ha-}{ }^{1}$ en condición de monocultivo durante la época seca. Al comparar la parte comestible de ambos pastos P. máximum cv Tanzania supera en un $42.8 \%$ a H. rufa.

Al comparar la cantidad de fitomasa de la parte no comestible respecto a la fitomasa total de los pasto en ambas épocas, se encontró que $P$. maximun $c v$ Tanzania pasó de $31.48 \%$ en invierno a $36.48 \%$ en verano y en el caso de H. rufa de $16.14 \%$ a $22.14 \%$; en las dos pasturas se muestra 
un ligero aumento de 5 y $6 \%$ respectivamente. Con base a lo anterior se demuestra, por diferencia, que la parte comestible en $H$. rufa fue mayor que $P$. maximun cv Tanzania (cuadro 2).

Durante el periodo lluvioso, la disponibilidad de biomasa total promedio del asocio $P$. maximun $c v$ Tanzania con leguminosas arbóreas fue de 4,6 t MS ha- ${ }^{1}$ superando en más del $23 \%$ a $H$. rufa. Este dato coincide con lo planteado por Verdacea et al (2008) quien estimó, en este mismo periodo $3.4 \mathrm{t} \mathrm{MS} \mathrm{h}^{-1}$. Durante el periodo seco, los pastos en ambas asociaciones experimentaron una reducción en la disponibilidad de biomasa (H. rufa 65\% y P. maximun cv Tanzania $71 \%$ ) respecto a la época lluviosa (cuadro 2 ).

En relación a la cantidad promedio de biomasa total de las leguminosas arbóreas aportada al sistema en los siete ciclos de pastoreo, L. leucocephala superó en la pastura Tanzania (296.7 kg de MS ha- ${ }^{1}$ ) en más del 106\% y en $H$. rufa (211.9 kg de MS ha- ${ }^{1}$ ) en un 7.2\% a G. sepium. Considerando que es un aporte complementario la biomasa de la leguminosa arbórea, la cantidad estimada promedio es inferior a los reportados por otro estudios de bloques compactos de leucaena donde se estima hasta una disponibilidad de $1795.98 \mathrm{~kg}$ de MS ha- ${ }^{1}$ corte-1 con frecuencia de corte de 49 días y con riego (González et al., 2003).

En relación a la cantidad de material aprovechable (Hojas y tallos tiernos) contenido en la biomasa total, $L$.

leucocephala man-

tiene la misma tendencia de superioridad en la pastura $P$. maximun cv Tanzania con $72.8 \%$ más que $G$. sepium, pero en la pastura H. rufa, G. sepium es superior $2.8 \%$ a L. leucocephala. En la Pastura H. rufa, L. leucocephala también superó a $G$. sepium en porcentaje menor de $7.2 \%$, destacando en esto, que los promedios de sobrevivencia son similares entre ambas especies, aunque en lo referido a cantidad de plantas por hectárea según diseño y distribución de las leguminosas arbóreas, G. sepium presentó 172 plantas más que $L$. leucocephala.

Al comparar la proporción de tallos leñosos en la biomasa total, ésta varió de acurdo a la época y sitio de pastura. Para el caso de L. leucocephala, en la pastura $P$. maximun cv Tanzania, ésta pasó de 30,7\% en época lluviosa a 32,1\% en la época seca, mientras que $G$. sepium pasó de $7,7 \%$ a $21.6 \%$. Igual comportamiento se obtuvo en la pastura $\mathrm{H}$. rufa donde L. leucocephala pasó de $21.6 \%$ a $25.6 \%$ y G. sepium de $14.6 \%$ a $17.5 \%$ en los periodos respectivos. Estos datos difieren a lo estimado por Toral et al., (2006) quien encontró que ambas leguminosas obtuvieron más del $40 \%$ en tallos leñosos en ambas épocas en plantaciones con 1 año de establecimiento (cuadro 2).
El aporte potencial de la biomasa aprovechable (hojas + tallos tiernos) de las leguminosas arbóreas en ambas pasturas osciló entre el 14 y el 15\%. En la pastura $P$. maximun cv Tanzania, L. leucocephala aportó e1 9.25\% y G. sepium $5.35 \%$ para un total del $14.6 \%$ y en $H$. rufa, L. leucocephala aportó el $7.69 \%$ y $G$. sepium el $7.91 \%$ para un total de $15.61 \%$. En promedio L. leucocephala en las dos pasturas registró un total de 1149 plantas ha-1 que equivale a un 52\% de sobrevivencia y G. sepium 1204 plantas ha- ${ }^{1}$ y una sobrevivencia de $46.5 \%$ (cuadro 2 ).

Altura, diámetro de macolla de los pastos $H$. rufa $y \boldsymbol{P}$. maximun cv Tanzania. Durante el período de estudio la pastura $H$. rufa presentó la mayor altura promedio de la macolla con $95,4 \mathrm{~cm}$, superando en más de $11 \%$ a $P$. máximum $\mathrm{cv}$ Tanzania. Lara (2005) determinó una altura en $P$. máximum cv Tanzania a los 84 días de establecido de $96 \mathrm{~cm}$, siendo menor en comparación con los otros pastos de ese estudio. No obstante, este pasto superó en diámetro de macolla en más de $16,5 \%$ respectivamente a $H$. rufa (cuadro 3 ).
Cuadro 3. Altura y diámetro de macolla

\begin{tabular}{|c|c|c|c|c|c|c|c|c|}
\hline \multirow[b]{2}{*}{$\begin{array}{l}\text { Ciclo de } \\
\text { Pastoreo }\end{array}$} & \multicolumn{4}{|c|}{ Pastura H. rufa } & \multicolumn{4}{|c|}{ Pastura P. máximum cv Tanzania } \\
\hline & Fecha & D D (días) & Altura $(\mathrm{cm})$ & $\begin{array}{l}\text { D. macolla } \\
(\mathrm{cm})\end{array}$ & Fecha & D D (días) & Altura $(\mathrm{cm})$ & $\begin{array}{l}\text { D. macolla } \\
(\mathrm{cm})\end{array}$ \\
\hline I & $17 / 09 / 09$ & 48 & 56.5 & 62.0 & $21 / 09 / 09$ & 50 & 69.5 & 65.4 \\
\hline II & $11 / 11 / 09$ & 51 & 207.8 & 77.1 & $12 / 11 / 09$ & 48 & 108.6 & 98.6 \\
\hline III & $27 / 01 / 10$ & 46 & 68.2 & 50.9 & $28 / 01 / 10$ & 48 & 38.5 & 71.7 \\
\hline IV & $18 / 03 / 10$ & 48 & 49.4 & 40.2 & $19 / 03 / 10$ & 49 & 35.1 & 51.4 \\
\hline V & $11 / 05 / 10$ & 53 & 42.8 & 48.1 & $12 / 05 / 10$ & 53 & 39.6 & 60.6 \\
\hline VI & $07 / 07 / 10$ & 51 & 110.2 & 89.2 & $07 / 07 / 10$ & 52 & 160.5 & 100.4 \\
\hline VII & $31 / 08 / 10$ & 48 & 132.7 & 99.5 & $31 / 08 / 10$ & 49 & 144.4 & 95.5 \\
\hline Promedio / & iclo & 49.3 & 95.4 & 66.7 & & 49.9 & 85.2 & 77.7 \\
\hline
\end{tabular}


Cuadro 4. Composición botánica

\begin{tabular}{|c|c|c|c|c|c|c|c|c|c|c|c|c|c|}
\hline \multirow{3}{*}{$\begin{array}{c}\text { Ciclo / } \\
\text { Pastoreo }\end{array}$} & \multicolumn{7}{|c|}{ Pastura $H$. rufa } & \multicolumn{6}{|c|}{ Pastura P. máximum cv Tanzania } \\
\hline & \multirow{2}{*}{$\begin{array}{l}\% \text { Cob. } \\
\text { vegetal }\end{array}$} & \multicolumn{5}{|c|}{ Composición botánica (\%) } & \multirow{2}{*}{$\begin{array}{l}\% \text { Cob } \\
\text { pasto }\end{array}$} & \multirow{2}{*}{$\begin{array}{l}\% \text { Cob. } \\
\text { vegetal }\end{array}$} & \multicolumn{4}{|c|}{ Composición botánica (\%) } & \multirow{2}{*}{$\begin{array}{c}\% \text { Cob. } \\
\text { pasto }\end{array}$} \\
\hline & & Pasto & $\begin{array}{l}\text { Hoja } \\
\text { ancha }\end{array}$ & $\begin{array}{l}\text { Otros } \\
\text { pastos }\end{array}$ & Ciperáceas & Total & & & Pasto & $\begin{array}{l}\text { Hoja } \\
\text { ancha }\end{array}$ & $\begin{array}{c}\text { Otros } \\
\text { pastos }\end{array}$ & Total & \\
\hline I & 56.3 & 78.6 & 17.2 & 4.1 & 0.0 & 100 & 44.3 & 56.4 & 96.6 & 2.7 & 0.8 & 100 & 54.4 \\
\hline II & 87.9 & 88.3 & 11.4 & 0.4 & 0.0 & 100 & 77.6 & 76.2 & 98.8 & 1.2 & 0.0 & 100 & 58.1 \\
\hline III & 50.3 & 76.0 & 20.0 & 4.0 & 0.0 & 100 & 38.2 & 31.8 & 98.9 & 0.9 & 0.2 & 100 & 31.5 \\
\hline IV & 28.4 & 91.9 & 7.8 & 0.3 & 0.0 & 100 & 26.1 & 29.3 & 98.1 & 1.9 & 0.0 & 100 & 28.7 \\
\hline V & 31.5 & 92.0 & 8.0 & 0.0 & 0.0 & 100 & 29.0 & 52.3 & 95.8 & 4.2 & 0.0 & 100 & 50.1 \\
\hline VI & 97.8 & 91.7 & 7.2 & 1.1 & 0.0 & 100 & 89.7 & 97.4 & 90.3 & 3.5 & 6.2 & 100 & 88.0 \\
\hline VII & 89.7 & 87.8 & 12.1 & 0.0 & 0.1 & 100 & 78.8 & 86.5 & 97.8 & 1.6 & 0.6 & 100 & 85.0 \\
\hline Media & 63.2 & 86.8 & 11.9 & 1.3 & 0.0 & 100 & 54.8 & 61.4 & 96.6 & 2.3 & 1.1 & 100 & 59.3 \\
\hline
\end{tabular}

$\mathrm{DD}=$ Días de descanso, $\mathrm{Cob}=$ Cobertura.

Contenido de PC en pastos. En el contenido de PC en la biomasa de los pastos, $P$. maximun cv Tanzania presentó los mayores valores en las cuatro evaluaciones tanto en la parte comestible y no comestible, superando en promedio en más del $34 \%$ y $2.3 \%$ a $H$. rufa respectivamente, aunque en este último dato no hay diferencia estadística (cuadro 5). Estos datos son similares a los encontrados por Ramos de Oliveira et al., (2013) quienes estimaron a los 75 días después del trasplante un valor de PC de $6.25 \%$ en toda la biomasa aérea de la planta.

Cuadro 5. Contenido de proteina cruda en biomasa de los pastos

\begin{tabular}{lccccc}
\hline Pastoreo & Fecha & \multicolumn{2}{c}{ H. rufa } & \multicolumn{2}{c}{ P. máximum cv Tanzania } \\
& & PC & PnC & PC & PnC \\
\hline I & 17 y $18 / 09 / 09$ & $8.3 \mathrm{a}$ & $4.0 \mathrm{a}$ & $14.9 \mathrm{~b}$ & $4.8 \mathrm{a}$ \\
II & 09 y $11 / 11 / 09$ & $8.7 \mathrm{a}$ & $5.1 \mathrm{a}$ & $7.4 \mathrm{a}$ & $4.1 \mathrm{a}$ \\
III & $27 \mathrm{y} 28 / 01 / 10$ & $5.3 \mathrm{a}$ & $4.6 \mathrm{a}$ & $7.3 \mathrm{~b}$ & $4.3 \mathrm{a}$ \\
IV & $18 \mathrm{y} 19 / 03 / 10$ & $4.1 \mathrm{a}$ & $3.0 \mathrm{a}$ & $6.2 \mathrm{~b}$ & $4.0 \mathrm{~b}$ \\
\hline Promedio & & $6.6 \mathrm{a}$ & $4.2 \mathrm{a}$ & $8.9 \mathrm{~b}$ & $4.3 \mathrm{a}$ \\
\hline
\end{tabular}

$\mathrm{PC}=$ Parte comestible, $\mathrm{PnC}=$ Parte no comestible

Letras distintas indican diferencias significativas, según LSD-Fischer $(p<=0,05)$.

En relación al efecto de la época sobre el contenido de la proteína cruda $H$. rufa su valor proteico fue superior en la época lluviosa con relación a la seca en más del $80 \%$ en la parte comestible y $98 \%$ en la parte no comestible, la misma tendencia presentó $P$. maximun $c v$ Tanzania en la parte comestible $(63.9 \%)$ donde la diferencia fue más marcada que en la parte no comestibles (7.2\%), aunque con menor diferencia porcentual en relación a $H$. rufa.

Contenido de PC en leguminosas arbóreas. L. leucocephala presentó los mayores valores de PC en las hojas en las cuatro evaluaciones superando en más del 13 y $18 \%$ a G. sepium en la pastura $P$. maximun cv Tanzania e H. rufa respectivamente; en

relación al contenido de $\mathrm{PC}$ en los tallos tiernos sucede lo contrario, en la pastura $H$. rufa la $L$. leucocephala contiene $4 \%$ más de PC que G. sepium, sin embargo esta tendencia es diferente en la pastura $P$. maximun cv Tanzania (cuadro 6). Datos estimados por Wencomo (2012) en la biomasa forrajera de L. leucocephala son similares a este estudio, en la época lluviosa estimó $25.96 \%$ PC y en la época seca un $23.96 \%$ de PC.

El contenido de PC de las hojas fue mayor al de los tallos tiernos en las dos leguminosas arbóreas, para el caso de L. leucocephala tanto en la pastura $H$. rufa y $P$. maximun cv Tanzania, el valor de la PC de las hojas superó a los tallos tiernos en más de 105 y 116\% respectivamente y para $G$. sepium esta diferencia fue de $81 \%$ en H. rufa y $70 \%$ en $P$. maximun cv Tanzania (cuadro 6).

El contenido de PC en ambas leguminosas varió conforme la época. Para L. leucocephala en la época lluviosa fue superior en más del $9 \%$ en las hojas y $32.7 \%$ en los tallos tiernos en la Pastura $H$. rufa respecto a la época seca, y en la pastura $P$. maximun cv Tanzania correspondió a un 19.8 y $50 \%$ en hojas y los tallos tiernos respectivamente; esta misma tendencia se observó en G. sepium en ambas pasturas. Esta tendencia coincide con lo planteado por Verdecia et al., (2014) y Wencomo (2012) quienes afirman que durante la época lluviosa la PC es mayor en relación a la época de poca lluvia (cuadro 6).

Cuadro 6. Contenido de proteína cruda en biomasa de los pastos

\begin{tabular}{|c|c|c|c|c|c|c|c|c|c|}
\hline \multirow{3}{*}{ 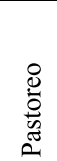 } & \multirow[t]{3}{*}{ Fecha } & \multicolumn{4}{|c|}{ Pastura $H$. rufa } & \multicolumn{4}{|c|}{ Pastura P. máximum cv Tanzania } \\
\hline & & \multicolumn{2}{|c|}{ L. leococephala } & \multicolumn{2}{|c|}{ G. sepium } & \multicolumn{2}{|c|}{ L. leococephald } & \multicolumn{2}{|r|}{ G. sepium } \\
\hline & & Hojas & $\begin{array}{l}\text { Tallos } \\
\text { tiernos }\end{array}$ & Hojas & $\begin{array}{l}\text { Tallos } \\
\text { tiernos }\end{array}$ & Hojas & $\begin{array}{l}\text { Tallos } \\
\text { tiernos }\end{array}$ & Hojas & $\begin{array}{l}\text { Tallos } \\
\text { tiernos }\end{array}$ \\
\hline I & 17 y $18 / 09 / 09$ & $25.9 \mathrm{a}$ & $14.8 \mathrm{a}$ & $23.3 \mathrm{a}$ & $14.9 \mathrm{a}$ & $26.6 \mathrm{~b}$ & $14.8 \mathrm{a}$ & $20.5 \mathrm{a}$ & $14.5 \mathrm{a}$ \\
\hline II & 09 у $11 / 11 / 09$ & $26.2 \mathrm{a}$ & $12.8 \mathrm{a}$ & $24.6 \mathrm{a}$ & $13.9 \mathrm{a}$ & $25.4 \mathrm{a}$ & $11.5 \mathrm{a}$ & $26.6 \mathrm{a}$ & $15.6 \mathrm{~b}$ \\
\hline III & 27 y $28 / 01 / 10$ & $26.1 \mathrm{~b}$ & $10.2 \mathrm{a}$ & $19.0 \mathrm{a}$ & $9.8 \mathrm{a}$ & $22.1 \mathrm{~b}$ & $8.0 \mathrm{a}$ & $18.9 \mathrm{a}$ & $8.8 \mathrm{a}$ \\
\hline IV & 18 y $19 / 03 / 10$ & $21.5 \mathrm{a}$ & $10.6 \mathrm{a}$ & $17.5 \mathrm{a}$ & $7.7 \mathrm{a}$ & $21.3 \mathrm{~b}$ & $9.6 \mathrm{a}$ & $17.7 \mathrm{a}$ & $10.3 \mathrm{a}$ \\
\hline \multicolumn{2}{|c|}{ Promedio } & $24.9 \mathrm{~b}$ & $12.1 \mathrm{a}$ & $21.1 \mathrm{a}$ & $11.6 \mathrm{a}$ & $23.8 \mathrm{~b}$ & $11.0 \mathrm{a}$ & $20.9 \mathrm{a}$ & $12.3 \mathrm{a}$ \\
\hline
\end{tabular}




\section{CONCLUSIONES}

Hyparrhenia rufa en este estudio alcanzó mayor altura promedio que Panicum máximum cv Tanzania, sin embargo esta última tiene mayor diámetro de macollas.

La cobertura vegetal de Panicum máximum cv Tanzania, según composición botánica, fue más alta que Hyparrhenia rufa, característica que le permitió tener menos presencia de otras especies (arvenses).

La inclusión de las leguminosas en el área de los pastos (28 a 30\%) permite mejorar de forma complementaria la disponibilidad de biomasa en las pasturas.

La disponibilidad de biomasa promedio de Panicum maximun cv Tanzania, fue superior a Hyparrhenia rufa en las dos épocas. Es importante mencionar que la parte no comestible (hojas secas y tallos lignificados) de este mismo pasto durante la época seca tiende a ser más consumida durante el pastoreo por los animales.
Leucaena leucocephala en asocio con Panicum maximun cv Tanzania e Hyparrhenia rufa presentó los valores más altos en biomasa y contenido de proteína cruda que Gliricidia sepium en esta misma asociación.

El contenido de la proteina cruda en los componentes del sistema varió según la época del año, denotándose mayores valores en la época lluviosa con respecto a la seca. En las partes comestibles de los pastos el contenido de proteína cruda fue mayor y en las leguminosas arbóreas el mayor valor se registró en las hojas.

Panicum maximun cv Tanzania y Leucaena leucocephala presentaron los mayores valores en biomasa y proteína cruda en el sistema silvopastoril "pastura en callejones".

\section{REFERENCIAS BIBLIOGRÁFICAS}

Giraldo, L; Bolívar D. 1999. Evaluación de un sistema silvopastoril de Acacia decurrens asociada con pasto kikuyo (Pennisetum clandestinum), en clima frío de Colombia. In Congreso Latinoamericano de agroforestería para la producción animal sostenible. 1ra Ed. 1999, Cali, Colombia. Memorias (en línea). Consultado el 23 de abr. de 2005. Disponible en: http://www.cipav.org.co/ redagrofor/memorias99/Memorias.htm

Hargreaves, JN; Kerr, JD. 1992. BOTANAL-A comprehensive sampling and computing procedure for estimating pasture yield and composition. 2. Computational package. Tropical Agronomy Technical Memorandum No. 79. CSIRO, Canberra, Australia. 83 p.

Haydock, KP; Shaw, NH. 1975. The comparative method for estimating dry matter yield pasture. Australian Journal of Experimental Agriculture and Animal Husbandary. 15:169-171.

INETER (Instituto Nicaragüense de Estudios Territoriales). S.f. Característica del clima de Nicaragua. Normas históricas-altas climático: 1971 -2000. (en línea). Consultado 20 oct. 2015. Disponible en http://webserver2.ineter.gob.ni/Direcciones/meteorologia/ clima\%20nic/caracteristicasdelclima.html

McDowell, LR; Conrad, JH; Ellis, GL; Loosli, JK. 1984. Minerales para rumiantes en pastoreo en regiones tropicales. Departamento de Ciencia Animal Centro Animal, Centro de Agricultura Tropical, Universidad de Florida, Gainesville y La Agencia de los Estados Unidos para el Desarrollo Internacional. 90 p.

Morales, J; Quemé, JS; Melgar, M. 2009. InfoStat, Manual de uso: Ejemplo de los principales métodos estadísticos utilizados en la industria cañera. Centro guatemalteco de investigación y capacitación de la caña de azúcar (CENGICAÑA). Santa Lucía de Cotz. $48 \mathrm{p}$.

Pezo, D; Ibrahim, M. 1999. Sistemas silvopastoriles: Módulo de enseñanza agroforestal № 2. 2da edición. Turrialba, CR. CATIE/ GTZ. 275 p.

Ramos de Oliveira, FL; Alves, MV; Soares RM; Tuffi SL; Faria de Oliveira, NJ; Castro, Gl. 2013.

Romero, N. 1997. Métodos de análisis para la determinación de nitrógeno y constituyentes nitrogenados en alimentos (Capitulo 15). In Producción y manejo de datos de composición química de alimentos en nutrición. FAO, Roma, IT. 356 p.

Salas, JB. 1993. Árboles de Nicaragua. Managua, Instituto Nicaragüense de Recursos Naturales y del Ambiente (IRENA). 390 p.

Spain, J.M; Gualdrón, R. 1991. Degradación y rehabilitación de pastures. In. Lascano, C:E; Spain. eds. Establecimiento y renovación de pastures, In. VI Reunión del Comité Asesor de la Red Internacional de Evaluación de Pastos Tropicales (RIEPT). Centro Internacional de Agricultura Tropical (CIAT). 283 p.

Stocking, M; Murnaghan, N. 2001. Handbook for the field assessment of land degradation. Earthscan Publications Ltd. Londres

Szott, L; Ibrahim, M; Beer, J. 2000. The hamburger connection hangover: cattle, pasture land use in Central America. Turrialba, Costa Rica. 71p.

Toral, O; Iglesias, J; Reino, J. 2006. Comportamiento de germoplasma arbóreo forrajero en condiciones de Cuba. Estación experimental de pasto y faorraje Indio Hatuey Central España Republicana CP 44280. Matanza, Cuba.

Tothill, JC; Hargreaves, JNG; Jones, RM; McDonald, CK. 1992. A comprehensive sampling and computing procedure for estimating pasture yield and composition. Queensaland, Australia, CSIRO. 23 p.

Verdecia, ADM; Herrera, GRS; Ramírez, JL; Acosta, IL; Bodas R, R; Lorente, SA; Giráldez GFJ. 2014. Caracterización bromatológica de seis especies forrajeras en el Valle del Cauto, Cuba. Avances en Investigación Agropecuaria. 2014. 18(3): 75-90.

Wencomo, H. 2012. Comportamiento de la disponibilidad de biomasa y la composición química en 23 accesiones de Leucaena spp. Matanza, Cuba. 15 p. 